\title{
A circular polarizer for the region of windowless vuv radiation
}

\author{
T Döhring, G Schönhense and U Heinzmann \\ Universität Bielefeld, Fakultät für Physik, D-4800 Bielefeld 1, Germany
}

Received 8 March 1991, accepted for publication 10 September 1991

\begin{abstract}
A circular polarizer for the region of windowless vuv is described. The device consists of two four-mirror assemblies, using reflectivity differences and phase shifts at gold surfaces known from the Fresnel equations. Owing to its variable angle arrangement the polarizer can be used between $30 \mathrm{~nm}$ and $120 \mathrm{~nm}$. When operated with Hel light $(58.43 \mathrm{~nm})$, a degree of circular polarization of more than $96 \%$ was achieved. The optical constants of gold have been determined by use of the transmitted intensity and polarization of the radiation.
\end{abstract}

\section{Introduction}

Due to the lack of transmitting materials in the spectral region of photon energies higher than $12 \mathrm{eV}$ one has to use physical effects other than birefringence or dichroism to produce circularly polarized light. One method is to use the circularly polarized out-of-plane synchrotron radiation (Heinzmann et al 1982, Schäfers et al 1986) or helical wigglers (Kincaid 1977) and double undulators (Kim 1984) at storage rings. Another method to obtain circularly polarized vUv up to about $72 \mathrm{~nm}$ is the nonlinear mixing of polarized laser radiation (Hilbig and Wallenstein 1983, Huth-Fehre et al 1990).

The aim of this work was to build a circular polarizer in connection with a standard vuv light source and a corresponding circular analyser for the windowless vUv region. The effect we used was the difference of the reflectivities for s- and p-polarized light and the phase shift between these components while reflecting light on a metal surface as can be calculated from the Fresnel equations.

The use of this effect was proposed by Johnson and Smith (1983) to convert synchrotron radiation from linear to circular polarization. We take a four-mirror reflection set-up as linear polarizer (Hunter 1978) and a similar four-mirror phase retarder to obtain circularly polarized radiation. Although being tested and characterized at the HeI wavelength $(58.43 \mathrm{~nm})$, the polarizer is designed to cover a wide photon energy range from approximately $10 \mathrm{eV}$ up to $40 \mathrm{eV}$. This is achieved by means of a variable-angle arrangement.

There is a variety of applications for circular polarizers and circularly polarized light. They may include spin-resolved photoemission studies (Heinzmann 1990), circular dichroism measurements (Allen and Bustamente 1985, and references therein), circular dichroism in photoemission (Schönhense 1990), determination of optical constants by ellipsometry (Johnson et al 1989) and the use as a circular analyser (Gaupp and Mast 1989) for circularly polarized vUV radiation from synchrotrons, undulators or sources of coherent vUV.

\section{Design and experimental performance}

\subsection{Experimental set-up}

When building a circular polarizer for initially unpolarized light one has first to produce linear polarized light and then use a quarter-wave retarder to induce the phase shift essential for the circular polarization. Furthermore, the quarter-wave retarder has to be rotated with respect to the linear polarizer in order to optimize the degree of circular polarization. On the other hand, the state of polarization behind the circular polarizer must be analysed. For this purpose we take the same arrangement, a quarter-wave retarder and a linear polarizer, now acting as an analyser.

The experimental set-up is illustrated in figure 1. It consists of the following assembly groups: windowless rare-gas discharge source, rotatable linear polarizer, phase retarder, second phase retarder, linear analyser, photocathode and channeltron detector. The second phase retarder and the linear analyser constitute a circular analyser having the same design parameters as the circular polarizer. Behind each optical element a transmitting photocathode (fine molybdenum mesh) or alternatively a simple polarization monitor (Rabinovitch et al 1965) can be inserted into the optical path for beam diagnostics.

The windowless rare-gas resonance lamp was an improved version of the type described by Schönhense and Heinzmann (1983). This version is fully bakeable up to $200^{\circ} \mathrm{C}$ and can be operated with either one or two differential pumping stages, the latter for UHV applications. The discharge capillary has a $2 \mathrm{~mm}$ bore of 


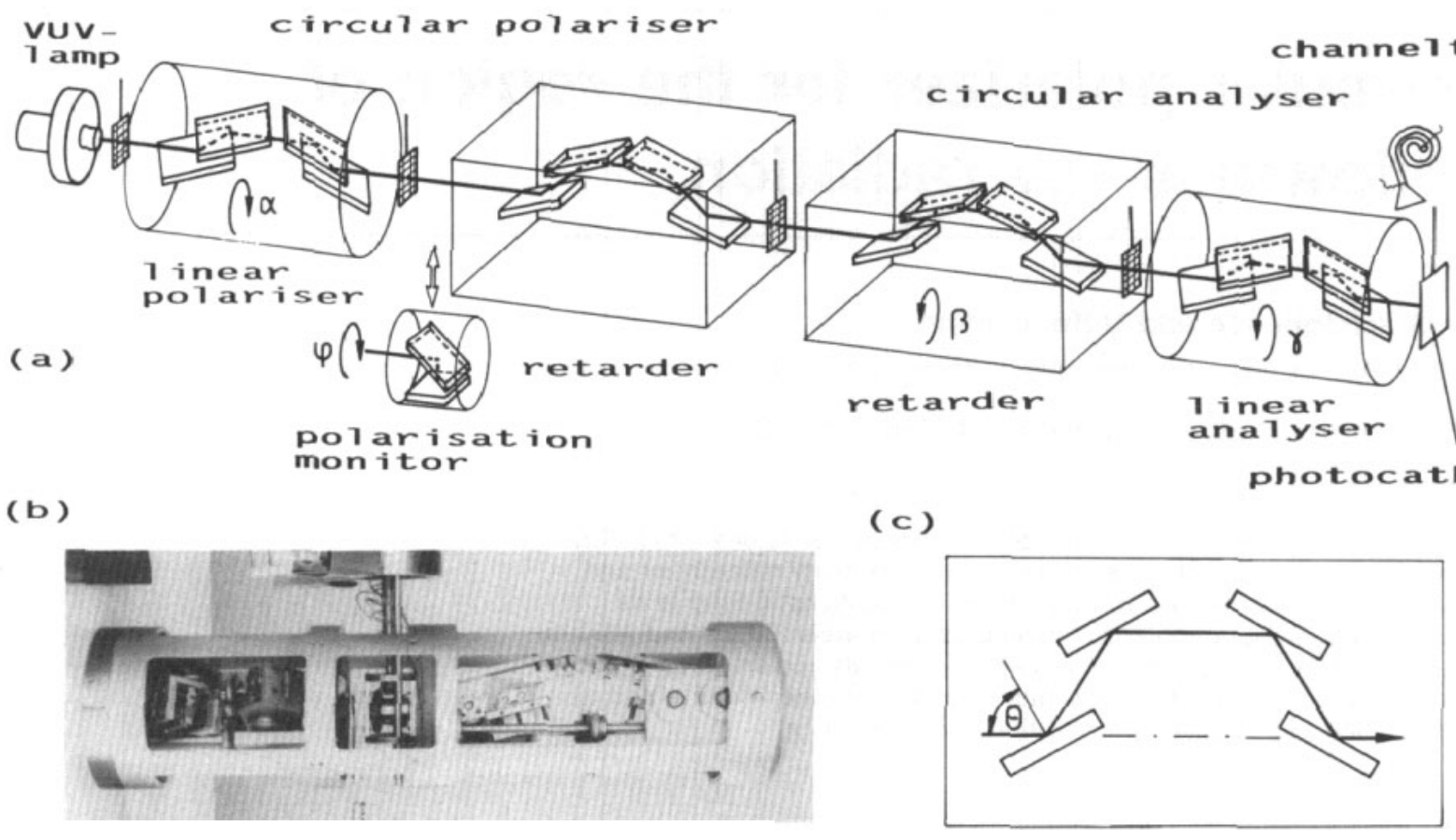

Figure 1. Experimental arrangement. (a) Schematic diagram of polarizer and analyser; (b) photograph of the circular polarizer; (c) optical path through one element.

$60 \mathrm{~mm}$ length and differential pumping is provided through a Pyrex capillary; for the present study a $2.2 \mathrm{~mm}$ bore and $200 \mathrm{~mm}$ length was employed. The maximum photon flux of the lamp is $10^{13}$ photons $\mathrm{s}^{-1}$.

Because of the strong influence of surface contamination on the polarizing quality noticed in earlier experiments (Schönhense 1980) the whole set-up was installed in an ultra-high-vacuum chamber. For pumping the continuous gas flow from the lamp a turbo-molecular pump $\left(1701 \mathrm{~s}^{-1}\right)$ was used. The base pressure was of the order of $10^{-7}$ mbar, the helium partial pressure during lamp operation was less than $10^{-5} \mathrm{mbar}$ (with one differential pumping stage only).

\subsection{Design of the polarizer}

The circular polarizer consists of two components, the linear polarizer and the phase retarder. In principle, it is possible to build a quarter-wave retarder with only one reflection (Westerveld 1985). In this case there is an angle of about $115^{\circ}$ between the incoming and outgoing light beams and, moreoever, this angle must be varied for optimization of the polarizer efficiency. For experimental applications, however, it is usually advantageous to have a coaxial set-up of ingoing and outgoing radiation. The minimum number of reflections is then three. For technical reasons we have chosen to construct a retarder with four mirrors. With this set-up it is simple to derive the phase shift of a single reflection.

In order to adjust the phase shift, $a$ fine and precise tuning of the angle of incidence is necessary. A schematic drawing for the mounting of the retarder and its set-up is shown in figure 2.
For changing the phase shift of the retarder, the angles of incidence of all four mirrors can be adjusted simultaneously under vacuum. The angle of incidence can be varied between $\theta_{\text {RET }}=70^{\circ}$ and $\theta_{R E T}=84^{\circ}$. An ultra-high-vacuum rotation feed through was coupled via copper bellows to a worm drive on the retarder. There the corresponding two worm gears act such that their shafts rotate in opposite directions. On each shaft there are two further worm drives mounted; the mirrors are coupled through a rigid support to the gears. The first and fourth mirrors rotate directly around the gear axis, the second and third mirrors rotate on an excentrical mount. This provides the variation of the angle of incidence between $\theta_{\mathrm{RET}}=70^{\circ}$ and $\theta_{\mathrm{RET}}=84^{\circ}$ without change of the optical path. Each mirror is clamped onto its support by a spring and its position is defined by three adjustment screws. Because each worm drive reduces the rotation by $1: 60$, the angle of incidence can be changed by only $0.1^{\circ}$ by one turn of the rotation feedthrough. To increase the precision of the drive, tension springs are used and all shafts are held by two ball bearings in a special mounting.

As mirror material a gold film of about $100 \mathrm{~nm}$ thickness evaporated on a microscope slide is used. The gold coating must have a smoothing effect on the surface roughness, because there was no significant influence of surface roughness on the reflectivities. Calculations using the optical constants of other metals show that retarders with mirrors with platinum, iridium or osmium coating may give about two or three times more intensity. However, the unknown change of reflectivity and phase shift by contamination let us choose gold which is probably the best material with regard to stability of its optical constants, owing to its inert behaviour. 


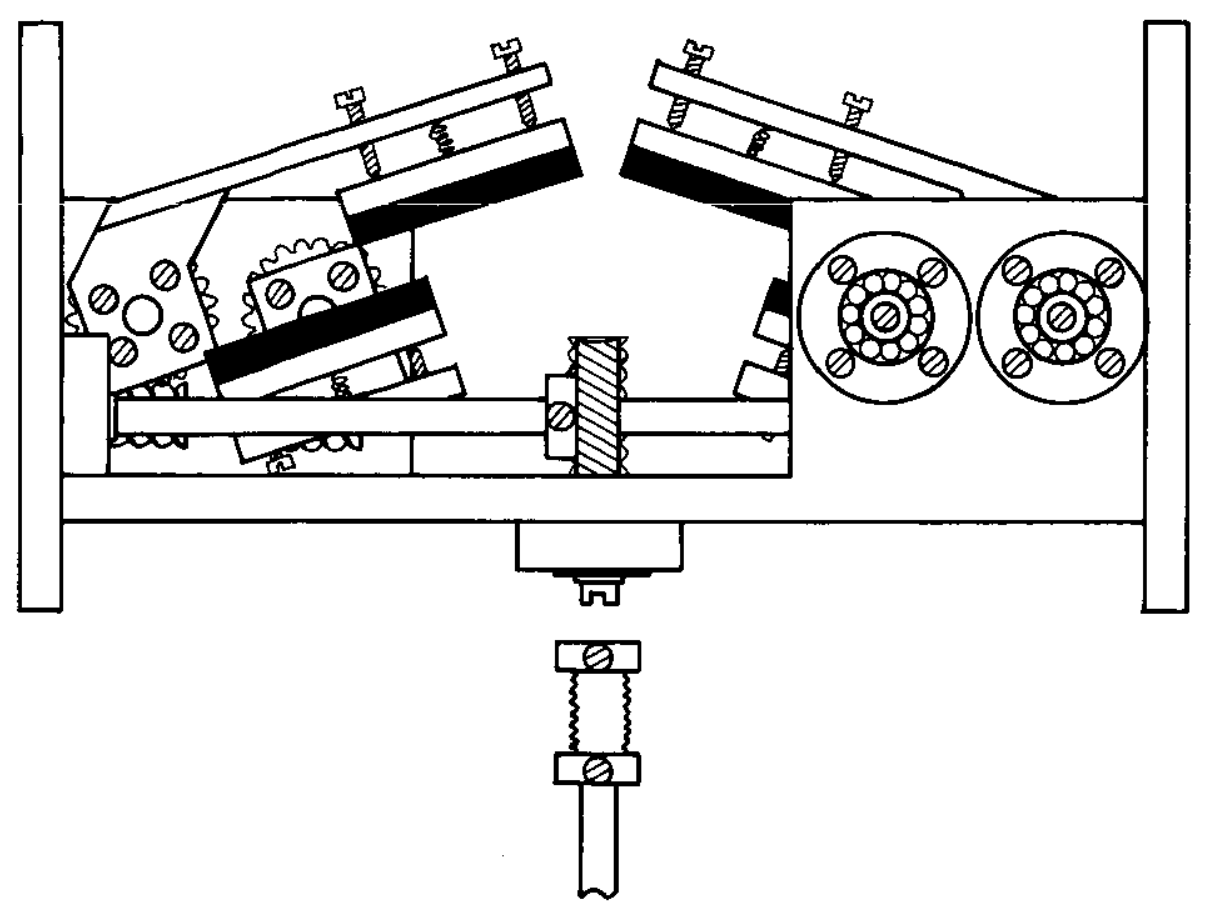

Figure 2. Schematic drawing of the phase retarder.

For the linear polarizer a similar four-mirror arrangement was utilized. Again, gold mirrors result in a high polarization and a good transmission; in order to obtain a high degree of polarization the angle of incidence must be about $60^{\circ}$ (Hunter 1978)

Being held by two large ball bearings and a bevelgear drive the whole linear polarizer can be rotated about the optical axis. To change the degree of polarization and the transmission of the linear polarizer, the angle of incidence can be varied between $\theta_{\mathrm{LP}}=50^{\circ}$ and $\theta_{\mathrm{LP}}=77^{\circ}$, without breaking the vacuum, using a drive similar to that of the retarder.

The complete unit of linear polarizer and retarder held by an optical bench mounting is shown in figure $1(b)$. By use of some small apertures the circular polarizer has an acceptance angle of $7 \mathrm{mrad}$, its total length is $430 \mathrm{~mm}$. Before being inserted into the vacuum chamber the complete set-up with sixteen mirrors was aligned on an optical bench employing a laser. After mounting in the vacuum housing the adjustment was checked by replacing the detector by a window with a fluorescent screen.

\subsection{Detection system}

For the purpose of vuv detection, a commercial photomultiplier turned out to be impracticable. The curved first dynode, which served as photocathode, caused a considerable polarization dependence. The detection efficiency varied with the relative angular orientation of the multiplier dynode to the electrical vector of the incoming radiation by more than $40 \%$. The fairly simple arrangement of the photocathode depicted in figure 1 overcame these difficulties. It consists of a planar copper photocathode of $50 \mathrm{~mm}$ diameter and a channeltron with amplifier and counting unit to detect the photoelectrons released from the cathode. Between photocathode and channeltron a voltage was applied to extract the electrons. This device showed a good linearity between light intensity and detector signal, independent of polarization and spatial beam fluctuations. To get an approximate absolute value of the photon flux, the photocurrent was measured directly with the copper photocathode by using a picoammeter.

Thin molybdenum meshes with an optical transmission of about $81 \%$ act as transmitting photocathodes in order to record the lamp intensity during measurement. The photocurrent was measured by means of a picoammeter. It was tested that the meshes do not influence the polarization.

\section{Measurements of the circular polarization}

For measurements of the circular polarization, the experimental set-up consisted of lamp, circular polarizer (i.e. linear polarizer and phase retarder), linear analyser and detector. Lamp, phase retarder and detector were kept fixed in space in the vacuum chamber and linear polarizer and linear analyser could be rotated.

For the determination of the photon helicity it is necessary to define the orientation of the rotation angles. The rotation angle $\alpha$ of the linear polarizer is defined as the angle between the plane of incidence of the phase retarder with respect to the plane of incidence of the linear polarizer (cf figure 1). The rotation angle $\gamma$ is defined in the same way as the angle between the plane of incidence of the linear analyser and the plane of 
incidence of the phase retarder. Note that in the laboratory system the retarder is fixed and $\alpha$ and $\gamma$ have opposite rotation directions. This way of definition follows the notation of the Müller rotation matrices (Hecht and Zajac 1979). In our experiment the intensity of left- and right-handed circularly polarized radiation was measured to be the same within the experimental uncertainty.

Each polar plot in figure 3 shows the intensity variation of the detected signal for rotation of the linear analyser at a certain set of parameters $\theta_{\mathrm{LP}}, \theta_{\mathrm{RET}}, \theta_{\mathrm{LA}}$ and $\alpha$. In this configuration $\theta_{\mathrm{LP}}$ was $65^{\circ} \pm 0.5^{\circ}$, a compromise between high degree of polarization and intensity, $\theta_{\mathrm{RET}}=$ $79.5^{\circ} \pm 0.5^{\circ}$ was optimized to get a phase shitt of neariy $\pi / 2$ and $\theta_{\mathrm{LA}}=60^{\circ}$ was chosen to get high accuracy in polarization analysis.

The set of polar plots shows the variation from totally 'senkrecht' linearly polarized light at an angle of $\alpha=0^{\circ}$ (i.e. the retarder acts as a second linear polarizer) over elliptically polarized light $\left(\alpha=60^{\circ}\right)$ to circularly polarized light at an angle of $\alpha=72^{\circ}$, indicated by the nearly circular polar plot. At this angle the linear polarization direction changes and at higher rotation angles the light is again elliptically polarized with 'parallel' linearly polarized components ( $\mathrm{cf} \alpha=90^{\circ}$ ).

From these intensity data the degree of circular polarization can be determined for each angle $\alpha$ as described by Heinzmann (1977), corrected for the linearly polarizing qualities of the retarder. The degree of circular polarization against rotation angle $\alpha$ is shown in figure 4 . The dots represent the measured values of $P_{\text {circ }}$, each corresponding to a polar plot as in figure 3 . The helicity of the circular polarization was taken from the comparison with values calculated by Müller matrices. The full
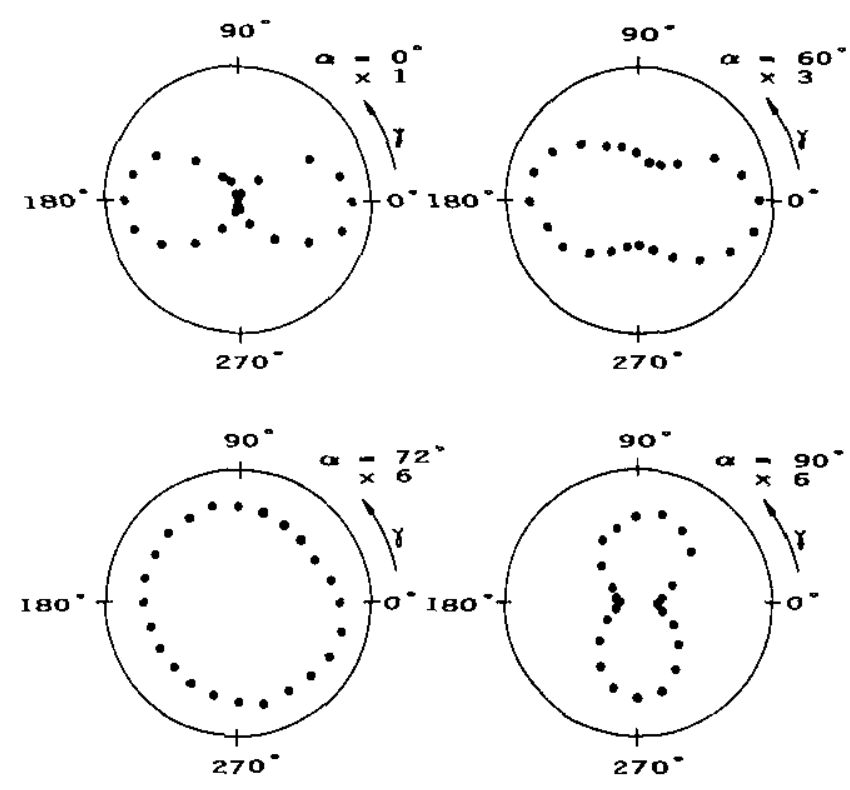

Figure 3. Polar plots showing the intensity variation at the detector while rotating the linear analyser with angle $\gamma$. The intensity scale at $\alpha=60^{\circ}$ is extended by a factor of three and at $\alpha=72^{\circ}$ and $90^{\circ}$ by a factor of six.

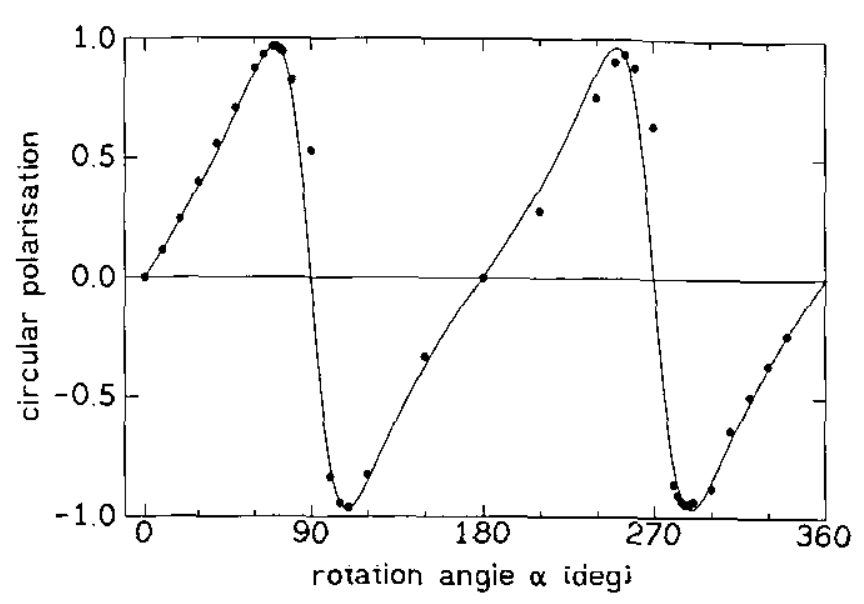

Figure 4. Degree of circular polarization against rotation angle $\alpha$. Note that the polarization reaches almost unity at certain angles.

curve symbolizes this calculation using the Fresnel equations based on $\varepsilon=0.40+\mathrm{i} \times 1.56$.

One can observe a change in light helicity from right-handed circular polarization at $\alpha=72^{\circ}$ and $\alpha=180^{\circ}+72^{\circ}$ to left-handed for $\alpha=180^{\circ}-72^{\circ}$ and $\alpha=360^{\circ}-72^{\circ}$ by simple rotation of the linear polarizer. The degree of circular polarization has been found to be higher than $96 \%$ in these positions. There is a small shift between calculated and measured values probably bccause of the backlash of the gears when the linear analyser was in the upside-down position.

In an extended set-up, a combination of a second $\pi / 2$ phase retarder and the linear analyser acts as a circular analyser (Gaupp and Mast 1989). This arrangement was shown in figure 1 . The measurement with this extended device showed that the second phase retarder converts the circular polarization back into a high linear polarization. Thus it was proven that behind the first retarder the light is clearly circularly polarized and not unpolarized, which could give the same results in the polar plots (figure 3 ).

The transmission of the circular polarizer as a function of rotation angle $\alpha$ is shown in figure 5. This measurement was made by comparing the photocurrent from the $\mathrm{Cu}$ cathode directly behind the lamp with the current from the same cathode placed behind the polarizer and corrected for aperture losses. A remaining estimated scaling error of $30 \%$ to $50 \%$ is due to different adjustment in both measurements. The measured transmission was $9 \times 10^{-4}$ for a rotation angle of $\alpha=72^{\circ}$. The full curve shows a fitted $\cos (2 \alpha)$ dependence because of the linearly polarizing property of the retarder.

The absolute transmission value for optimized circular polarization (at $\alpha=72^{\circ}$ ) was $2.7 \times 10^{-4}$ including losses due to apertures and transmission photocathodes.

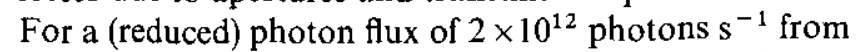
the lamp this corresponds to $6 \times 10^{8}$ photons $\mathrm{s}^{-1}$ with more than $96 \%$ degree of circular polarization.

For many applications of the polarizer in experiments it is not necessary to use the highest possible degree of 


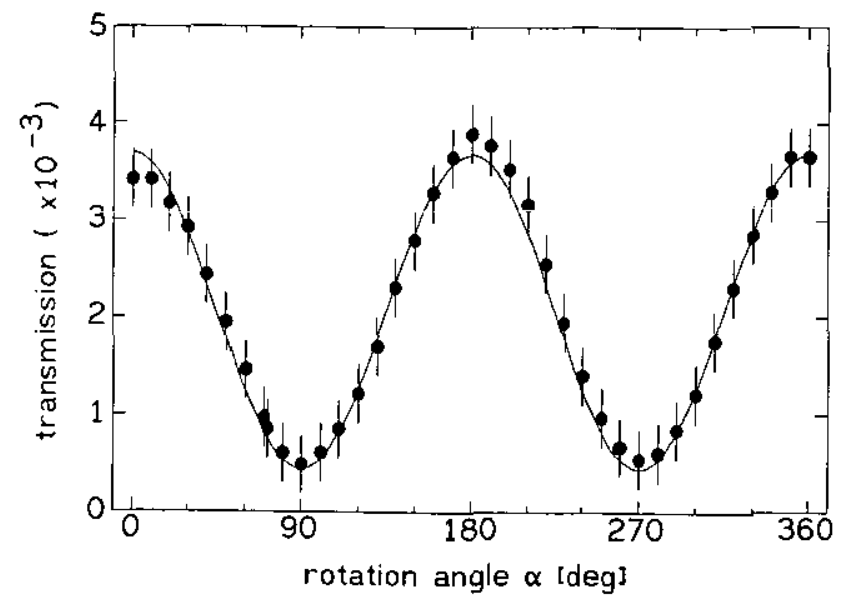

Figure 5. Transmission of the circular polarizer for unpolarized incident light against rotation angle $\alpha$.

circular polarization, because the optimization parameter of a polarizer or analyser is $\left(P_{\text {circ }}\right)^{2} \times I$. Thus the polarization can be reduced in order to gain higher intensity. This can be achieved by changing $\theta_{\mathrm{LP}}, \theta_{\mathrm{RET}}$ or the rotation angle $\alpha$. Other possibilities for an improvement of the transmission are a reduction of the aperture losses by using focusing mirrors or an increase of the reflectivities by use of other mirror materials, for example by using multilayer mirrors.

\section{Experimental determination of reflectivity and optical constants}

For a quantitative comparison of the experimental data with theory, based upon the Fresnel equations, one has to know the complex refractive index of the material at

- the photon energy used. There exist, however, several values for these constants in literature. They depend on the methods used for preparation and the model used in the evaluation of measured data. We determined the optical constants of the $100 \mathrm{~nm}$ evaporated gold coatings in the polarizer at $58.43 \mathrm{~nm}$ by inversion of the Fresnel equations. This requires measurement of the absolute transmission and the polarization power of the linear polarizer against the angle of incidence. For calibration of the linear polarizer, the rotation of a linear analyser with the same angle of incidence was performed in order to get the degree of polarization by the square root of the asymmetry factor in the intensity variation. Knowing the degree of polarization at a certain angle, the polarization versus angle of incidence can be measured by changing the angle of incidence of the polarizer. This dependence of polarization and transmission for the linear polarizer is shown in figure 6 .

In figure $6(a)$ the measured degrees of linear polarization of a four-mirror polarizer for unpolarized incident light are plotted (dots). Note that there is a large range of angles of incidence between $50^{\circ}$ and $70^{\circ}$ which leads to a high polarization. Figure $6(b)$ shows the measured transmission of the linear polarizer for unpolarized light

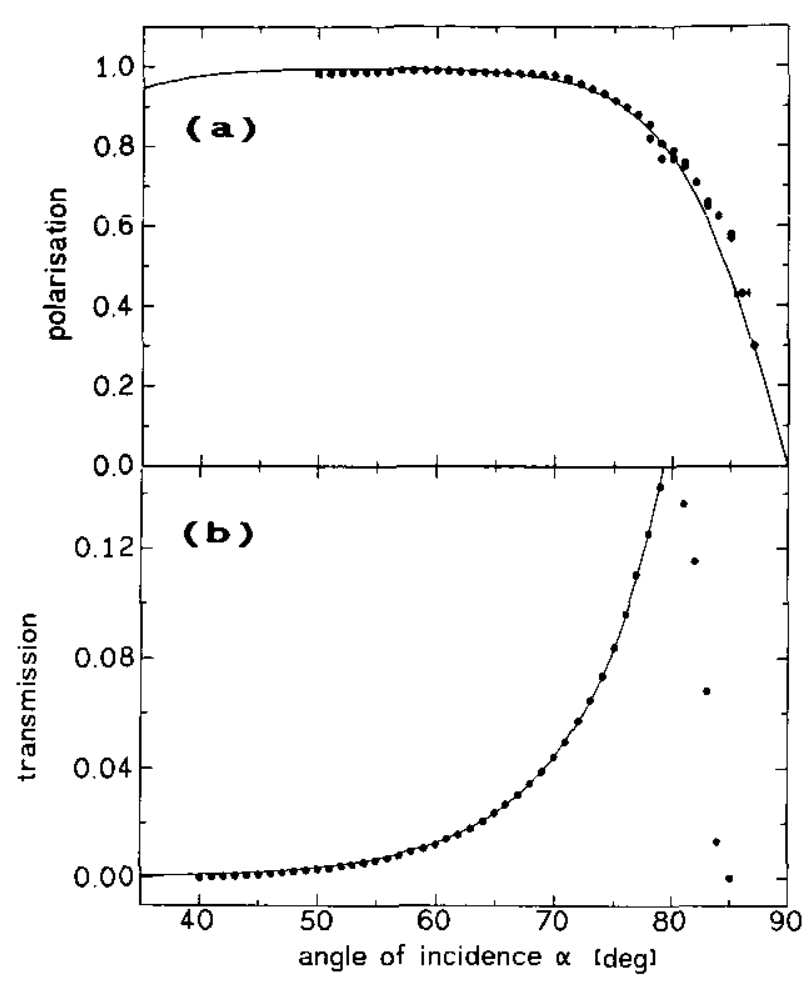

Figure 6. Polarization (a) and transmission (b) of a fourmirror linear polarizer with gold mirrors at the Hel resonance line $(58.43 \mathrm{~nm})$ for unpolarized incident light.

versus angle of incidence (dots). At the grazing incidence end of the angular scale the full aperture of the linear polarizer cannot be used and thus the transmitted intensity is decreased.

The measurements are compared with data calculated from the Fresnel equations based on $\varepsilon=0.40+\mathrm{i} \times 1.56$ (full curves) without correction for losses due to roughness of the surface.

The reflectivities for s- and p-polarized light can be calculated from the polarization and transmission data of the four-mirror polarizer according to

$$
\begin{aligned}
& R_{\mathrm{s}}=[T(1+P)]^{1 / 8} \\
& R_{\mathrm{p}}=[T(1-P)]^{1 / 8}
\end{aligned}
$$

with $T$ being the transmission and $P$ the linear polarization power of the polarizer. In figure $7(a)$ the reflectivities measured for 'senkrecht' linearly polarized light $R_{\mathrm{s}}$ and for 'parallel' linear polarization $R_{\mathrm{p}}$ are plotted. They are determined from the measurement of linear polarization and transmission of the four-mirror polarizer with variable angles of incidence. The experimental results (dots) are compared with calculated values using the Fresnel equations based on $\varepsilon=0.40+\mathrm{i} \times 1.56$ (full curves).

Deviation of the experimental points from the theoretical curves for angles of incidence below $60^{\circ}$ is most likely due to scattered unpolarized light. The phase shift derived from the circular polarization measurements (figure $7(b)$ ) is plotted for a single surface reflection, the four-mirror phase retarder has a phase shift of nearly $\pi / 2$. Knowing the data of $R_{\mathrm{s}}$ and $R_{\mathrm{p}}$ and the angle of incidence, it is possible to calculate the complex dielec- 


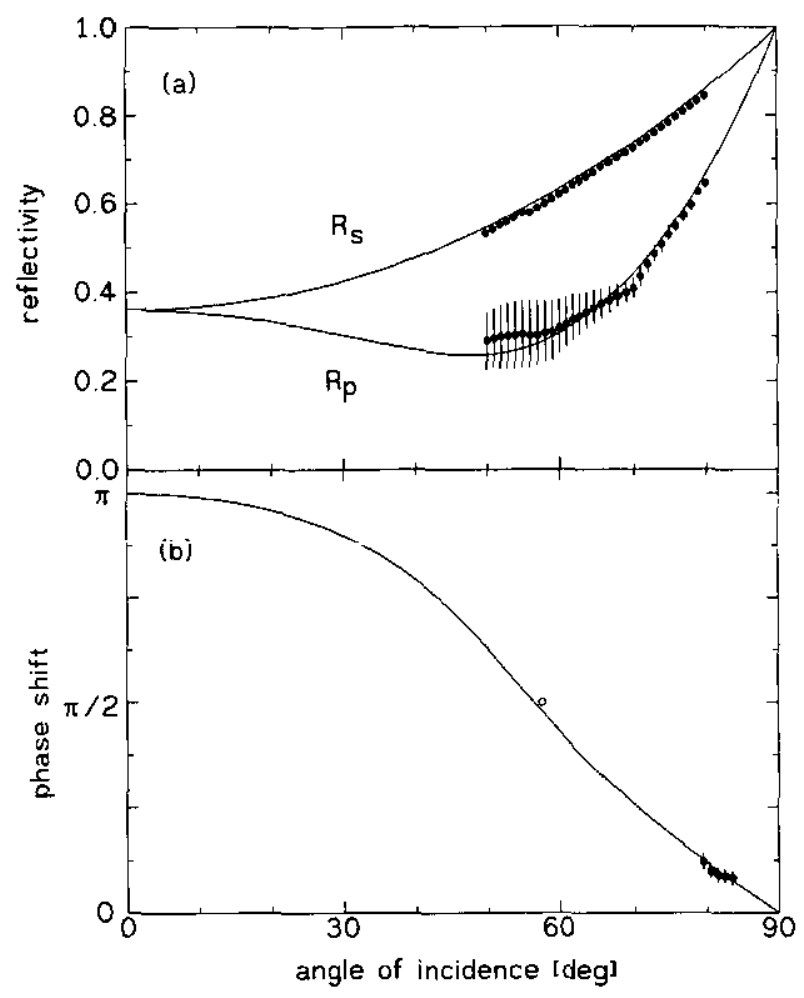

Figure 7. Reflectivities and phase shifts for gold mirrors at $58.43 \mathrm{~nm}$. (a) The reflectivities for s-polarized light $\left(R_{s}\right)$ and for p-polarization $\left(R_{\mathrm{p}}\right)$. (b) Phase shift between the $s$ and $\mathrm{p}$ reflected waves. These data for the four-mirror retarder (dots, this work) and for a one-mirror retarder (open circle, Westerveld et al 1985), are compared with the calculation (full curve).

trical constant by inversion of the Fresnel equations (Querry 1969). Average values of the dielectrical constants of $\varepsilon_{1}=0.40 \pm 0.03$ and $\varepsilon_{2}=1.56 \pm 0.05$ were determined for the gold films used. The deviations between our data and different values in the literature of $\varepsilon_{1}=0.33$ and $\varepsilon_{2}=1.86$ (Weaver et al 1981), $\varepsilon_{1}=0.33$ and $\varepsilon_{2}=1.80$ (Windt et al 1988) or $\varepsilon_{1}=0.64$ and $\varepsilon_{2}=1.52$ (Samson 1967) probably reflect the influence of the different preparation methods.

\section{Conclusions}

A circular polarizer for vUV radiation has been designed and investigated. This device consists of two four-mirror arrangements, one acting as a linear polarizer and one as a phase retarder. Gold films of about $100 \mathrm{~nm}$ thickness evaporated on microscope slides served as mirrors. By simultaneous variation of the angle of incidence, the wavelength optimization may be changed between $30 \mathrm{~nm}$ and $120 \mathrm{~nm}$. The circular polarizer was tested with HeI radiation from a windowless rare-gas discharge lamp. A second gold-mirror arrangement served as a circular analyser for characterization.

When operated with HeI light $(58.43 \mathrm{~nm})$, degrees of circular polarization of more than $96 \%$ have been measured for both helicities. By rotation of the linear polarizer the helicity of the light can be easily reversed. The whole circular polarizer has a transmission of $2.7 \times 10^{-4}$ with respect to an unpolarized incident $\mathrm{HeI}$ light beam, including aperture losses. With a (reduced) lamp intensity of $2 \times 10^{12}$ photons $\mathrm{s}^{-1}$, a photon flux of $6 \times 10^{8}$ photons $\mathrm{s}^{-1}$ has been obtained behind the polarizer with a degree of circular polarization of more than $96 \%$. For future investigations, the intensity might be increased by focusing mirrors, optimization of mirror materials or by reducing the degree of polarization by variation of the impact angles.

In addition, the polarization and the transmission of the linear polarizers were determined for different angles of incidence. From these measurements the reflectivities and the complex dielectrical constant of the gold mirrors used have been determined at a wavelength of $58.43 \mathrm{~nm}$.

It has been demonstrated that the polarizer may be a useful device to produce circularly polarized light for many experiments. It can also be used as an analyser to determine the degree of circular polarization of other sources.

\section{Acknowledgments}

We acknowledge invigorating discussions with Drs N Böwering, N Müller and A Gaupp. Support by SFB 216 (DFG) is gratefully acknowledged.

\section{References}

Allen F and Bustamente C. 1985 Applications of circulary polarized radiation using synchrotron and ordinary sources (New York: Plenum)

Gaupp A and Mast M 1989 First experimental experience with a vuv polarimeter at BESSY Rev. Sci. Instrum. 60 2213-5

Hecht E and Zajac A 1979 Optics (London: Addison Wesley)

Heinzmann U 1977 An apparatus for the production of circularly polarised vUV radiation $J$. Phys. E.: Sci. Instrum. 10 1001-5

Heinzmann U, Osterheld B and Schäfers F 1982 Measurements and calculations of the circular polarization and of the absolute intensity of synchrotron radiation in the wavelength range from 40 to $100 \mathrm{~nm}$ Nucl. Instrum. Methods 195 395-8

Heinzmann U 1990 Photoemission with circularly polarized photons Photoemission and Absorption Spectroscopy of Solids and Interfaces with Synchrotron Radiation eds M Campagna and R Rossi (Amsterdam: North-Holland) pp $469-88$

Hilbig R and Wallenstein R 1983 Resonant sum and difference frequency mixing in $\mathrm{Hg}$ IEEE $J$. Quant. Electron. 19 1759-70

Hunter W R 1978 Design criteria for reflection polarizers and analyzers in the vacuum ultraviolet Appl. Opt. 17 $1259-70$

Huth-Fehre T, Mank A, Drescher M, Böwering $\mathbf{N}$ and Heinzmann U 1990 Rotationally resolved Fano effect of HI molecules: An experimental study using coherent vacuum-ultraviolet radiation Phys. Rev. Lett. 64 396-9

Johnson P D and Smith N V 1983 Production of circularly polarized light from synchrotron radiation in the vacuum ultraviolet region Nucl. Instrum. Methods 214 505-8 
Johnson R L, Barth J, Cardona M, Fuchs D and Bradshaw A M 1989 Spectroscopic ellipsometry with synchrotron radiation Rev. Sci. Instrum. 60 2209-12

Kim K J 1984 A synchrotron radiation source with arbitrarily adjustable elliptical polarization $\mathrm{Nucl}$. Instrum. Methods 219 425-9

Kincaid B M 1977 A short-period helical wiggler as an improved source of synchrotron radiation J. Appl. Phys. $482684-91$

Querry M R 1969 Direct solution of the generalized Fresnel reflectance equations JOSA $59876-7$

Rabinovitch K, Canfield L R and Madden R P 1965 A method for measuring polarization in the vacuum ultraviolet Appl. Opt. 4 1005-10

Samson J A R 1967 Techniques of vacuum ultraviolet spectroscopy (New York: Wiley)

Schäfers F, Peatman W, Eyers A, Heckenkamp Ch, Schönhense $G$ and Heinzmann U 1986 High-flux normal incidence monochromator for circularly polarized synchrotron radiation Rev. Sci. Instrum. 57 1032-41
Schönhense G 1980 Angular dependence of the polarization of photoelectrons ejected by plane-polarized radiation from argon and xenon atoms Phys. Rev. Lett. 44 640-3

Schönhense G 1990 Circular dichroism and spin polarization in photoemission from adsorbates and non-magnetic solids Phys. Scr. T31 255-75

Schönhense G and Heinzmann U 1983 A capillary discharge tube for the production of intense vUV resonance radiation $J$. Phys. E: Sci. Instrum. 16 74-82.

Weaver J H, Krafka C, Lynch D W and Koch E E 1981 Optical properties of metals Physics Data Vol 182 (Fachinformationszentrum Karlsruhe)

Westerveld W B, Becker K, Zetner P W, Corr J J and McConkey J W 1985 Production and measurement of circular polarization in the vuv Appl. Opt. 24 2256-62

Windt D L, Cash W C, Scott M, Arendt P, Newnam B, Fischer R F and Swartzlander A B 1988 Optical constants for thin films of $\mathrm{Ti}, \mathrm{Zb}, \mathrm{Nb}, \mathrm{Mo}, \mathrm{Ru}, \mathrm{Rh}, \mathrm{Pd}$, $\mathrm{Ag}, \mathrm{Hf}, \mathrm{Ta}, \mathrm{W}, \mathrm{Re}, \mathrm{Ir}, \mathrm{Os}, \mathrm{Pt}$, and Au from 24 to $1216 \AA$ Appl. Opt. 27 246-78 